\title{
On the Security of the 128-Bit Block Cipher DEAL
}

\author{
Stefan Lucks ${ }^{\star}$ \\ Theoretische Informatik \\ University of Mannheim, 68131 Mannheim A5, Germany \\ lucks@th.informatik.uni-mannheim.de
}

\begin{abstract}
DEAL is a DES-based block cipher proposed by Knudsen. The block size of DEAL is 128 bits, twice as much as the DES block size. The main result of the current paper is a certificational attack on DEAL192, the DEAL variant with a 192-bit key. The attack allows a trade-off between the number of plaintext/ciphertext pairs and the time for the attacker's computations. Nevertheless, the DEAL design principle seems to be a useful way of doubling the block size of a given block cipher.
\end{abstract}

\section{Introduction}

The "data encryption standard" (DES) is the world's most well known symmetric cipher. Formally, the standard defines a 64 -bit key, but 8 bits are defined as "parity bits" and only 56 bits are actually used as the encryption key, i.e., the DES key size is 56 bits. Brute-force attacks for recovering a key are feasible, today - and considered the only practical way of breaking DES. Thus, while the DES itself cannot be considered secure, it is still attractive to use it as a component for designing another cipher with an increased key size, such as triple DES. A concern both for DES and for triple DES is the block size of only 64 bits, which may lead to matching ciphertext attacks.

In [1], Knudsen proposes the $r$-round Feistel cipher DEAL with a block size of 128 bits. It uses DES in the round function and accepts three different key sizes, namely 128, 192, and 256 bits. For the first two sizes, the author recommends $r=6$, for 256 bit keys, the number $r$ of rounds should be 8 . Depending on the key size, the three variants of DEAL are denoted DEAL-128, DEAL-192, and DEAL-256. DEAL is suggested as a candidate for the NIST AES standard.

This paper is organised as follows. In Section 2, a description of DEAL itself is given, Section 3 presents attacks on the six-round version of DEAL, and Section 4 deals with further concerns and conclusions.

* Supported by German Science Foundation (DFG) grant KR 1521/3-1. 


\section{A Description of DEAL}

Next, we describe the block cipher DEAL and the key schedules for DEAL-128, DEAL-192, and DEAL-256.

\subsection{The DEAL Core}

A 128-bit plaintext is split up into two halves $\left(x_{0}, y_{0}\right) \in\left(\{0,1\}^{64}\right)^{2}$. Two consecutive rounds $j$ and $j+1$ of DEAL take the 128-bit block $\left(x_{j-1}, y_{j-1}\right) \in\left(\{0,1\}^{64}\right)^{2}$ and the two round keys $R_{j}$ and $R_{j+1}$ as the input to compute the output block $\left(x_{j+1}, y_{j+1}\right) \in\left(\{0,1\}^{64}\right)^{2}$ by

$$
\begin{array}{ll}
x_{j}:=x_{j-1}, & y_{j}:=y_{j-1} \oplus E_{R_{j}}\left(x_{j-1}\right), \\
x_{j+1}:=x_{j} \oplus E_{R_{j+1}}\left(y_{j}\right), \quad \text { and } \quad & y_{j+1}:=y_{j},
\end{array}
$$

where $\oplus$ describes the bit-wise xor-operation for 64-bit strings and $j$ is odd. By $E$, we denote the DES encryption function. Two rounds $j$ and $j+1$ are also described in Figure 1.

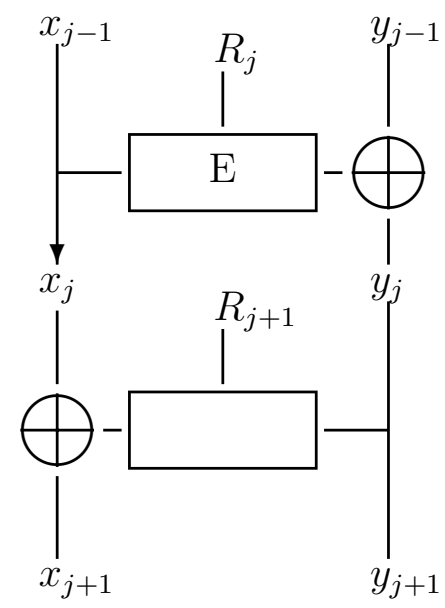

Fig. 1. Round $j$ and $j+1$ of DEAL, $j$ odd

Thus, for DEAL-128 and DEAL-192 we need 6 round keys $R_{1}, \ldots R_{6}$, for DEAL 256 we need 8 round keys $R_{1}, \ldots R_{8}$. Internally, every round key is used as a DES key, ignoring the "parity bits" and hence consists of 56 bits. We need three "key scheduling" algorithms to generate the round keys from the given master key of 128, 192, or 256 bits.

See Figure 2 for a visual description of six rounds of DEAL. 


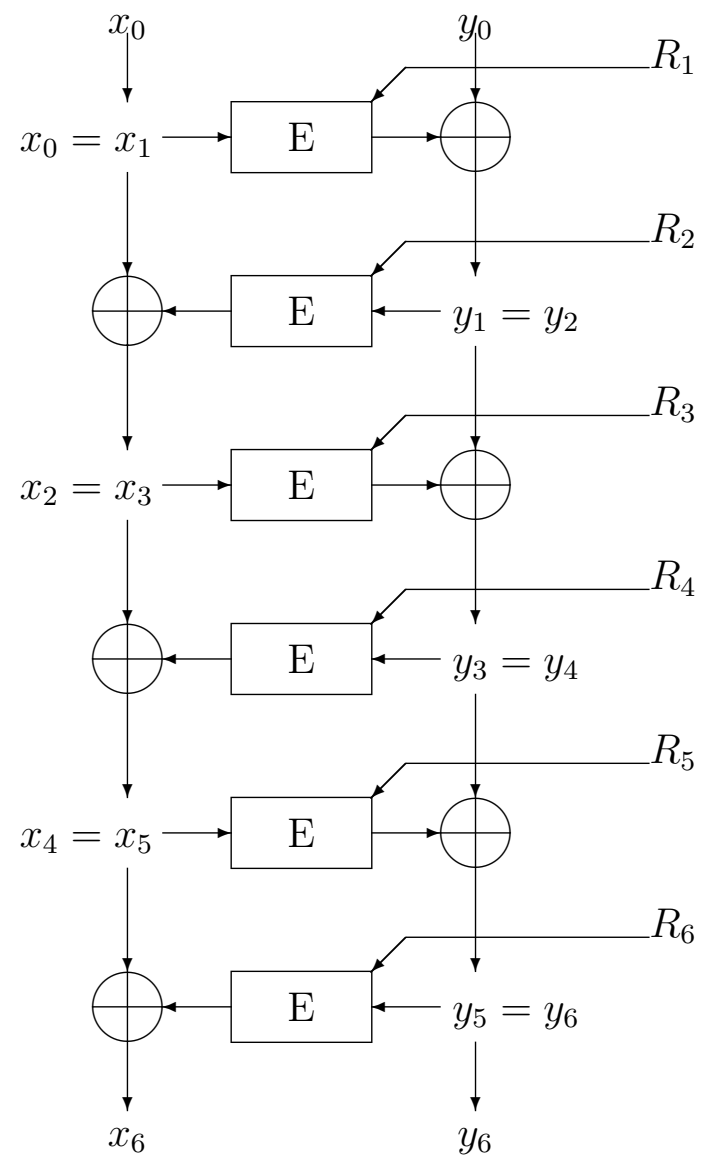

Fig. 2. The six-round version of DEAL

\subsection{The DEAL Key Schedule}

The key schedule of DEAL takes $s$ keys $K_{1}, \ldots, K_{s}$ of 64 bits each $(s \in$ $\{2,3,4\})$ and returns $r$ round keys $R_{1}, \ldots, R_{r}$ of 56 bits each $(r \in\{6,8\})$. The round keys are generated using DES encryption under a fixed DESkey $R_{*}$ (which is $R_{*}=0123456789$ abcdef in hexadecimal notation). 1 , $\ldots, \underline{4}$ are four different constant 64 -bit strings, where none is 000...0. The DEAL-128 round keys are generated from $K_{1}, K_{2}$ as follows:

$$
\begin{aligned}
& R_{1}:=E_{R_{*}}\left(K_{1}\right) \\
& R_{2}:=E_{R_{*}}\left(K_{2} \oplus R_{1}\right) \\
& R_{3}:=E_{R_{*}}\left(K_{1} \oplus R_{2} \oplus \underline{1}\right)
\end{aligned}
$$




$$
\begin{aligned}
& R_{4}:=E_{R_{*}}\left(K_{2} \oplus R_{3} \oplus \underline{2}\right) \\
& R_{5}:=E_{R_{*}}\left(K_{1} \oplus R_{4} \oplus \underline{3}\right) \\
& R_{6}:=E_{R_{*}}\left(K_{2} \oplus R_{5} \oplus \underline{4}\right)
\end{aligned}
$$

The DEAL-192 round keys are generated from $K_{1}, K_{2}$, and $K_{3}$ like this:

$$
\begin{aligned}
& R_{1}:=E_{R_{*}}\left(K_{1}\right) \\
& R_{2}:=E_{R_{*}}\left(K_{2} \oplus R_{1}\right) \\
& R_{3}:=E_{R_{*}}\left(K_{3} \oplus R_{2}\right) \\
& R_{4}:=E_{R_{*}}\left(K_{1} \oplus R_{3} \oplus \underline{1}\right) \\
& R_{5}:=E_{R_{*}}\left(K_{2} \oplus R_{4} \oplus \underline{2}\right) \\
& R_{6}:=E_{R_{*}}\left(K_{3} \oplus R_{5} \oplus \underline{3}\right)
\end{aligned}
$$

Given $K_{1}, K_{2}, K_{3}$, and $K_{4}$, the DEAL-256 round keys are:

$$
\begin{aligned}
& R_{1}:=E_{R_{*}}\left(K_{1}\right) \\
& R_{2}:=E_{R_{*}}\left(K_{2} \oplus R_{1}\right) \\
& R_{3}:=E_{R_{*}}\left(K_{3} \oplus R_{2}\right) \\
& R_{4}:=E_{R_{*}}\left(K_{4} \oplus R_{3}\right) \\
& R_{5}:=E_{R_{*}}\left(K_{1} \oplus R_{4} \oplus \underline{1}\right) \\
& R_{6}:=E_{R_{*}}\left(K_{2} \oplus R_{5} \oplus \underline{2}\right) \\
& R_{7}:=E_{R_{*}}\left(K_{3} \oplus R_{6} \oplus \underline{3}\right) \\
& R_{8}:=E_{R_{*}}\left(K_{4} \oplus R_{7} \oplus \underline{4}\right)
\end{aligned}
$$

The parity bits of the 64-bit values $R_{i}$ are ignored when $R_{i}$ is used as a DES-key (i.e., a DEAL round key), but relevant for computing $R_{i+1}$.

\section{Attacking DEAL}

Well known meet-in-the-middle techniques can be used to recover the DEAL round keys. This was stressed by Knudsen himself. The six-round version of DEAL is vulnerable to a meet-in-the-middle attack requiring roughly $\left(2^{56}\right)^{3}=2^{168}$ encryptions. For the eight-round version, the attack needs roughly $\left(2^{56}\right)^{4}=2^{224}$ encryptions. 
Thus the theoretical key size of DEAL is approximately no more than 168 for the six-round version and 224 for the eight-round version. This bounds the theoretical key size of DEAL-192 (six rounds) and DEAL-256 (eight rounds). Due to their memory requirements, these meet-in-themiddle techniques are quite unrealistic, though trade-off techniques to save storage space at the cost of increased running time are known [3].

Note that finding a DEAL- $n$ key by exhaustive key search (by "brute force" ) takes about $c * 2^{n} / 2$ single DES encryptions with $c=8$ for DEAL$192, c=9$ for DEAL-128, and $c=11$ for DEAL-256. (One can reject most combinations of round keys before the last round. I.e., rejecting a combination of round keys takes about 5 DES encryptions for the sixround variants of DEAL and about 7 DES encryptions for the eight-round variant. In the case of DEAL-192, we need to generate the first two round keys at most every $2^{64}$ steps, while the round keys $R_{3}, R_{4}$, and $R_{5}$ are generated every step. This takes about 3 DES encryptions. The reasoning for DEAL-128 and DEAL-256 is similar.)

\subsection{A Chosen Ciphertext Attack for the Six-Round Version}

In addition to meet-in-the-middle attacks, Knudsen describes a chosen plaintext attack to recover the round keys of the six-round version of DEAL. His attack requires about $2^{121}$ DES-encryptions using roughly $2^{70}$ chosen plaintexts. This is significantly faster than the average number of DES-encryptions needed to break DEAL-128 by exhaustive key search (i.e., $2^{127}$ encryptions) and greatly faster than the number of DESencryptions to break DEAL-192 $\left(2^{191}\right)$. Due to the huge amount of chosen plaintexts, Knudsen claims that exhaustive key search nevertheless is less unrealistic than this attack.

We will use the same technique, but we are going backwards, i.e., our attack is a chosen ciphertext attack to gain information about the first round key $R_{1}$. Given the first 56-bit round key $R_{1}$, there are only $2^{8}$ possible choices for the first sub-key $K_{1}$ of the master key. Knowing the last 56-bit round key instead of the first one, is somewhat less helpful for the attacker, due to the DEAL key schedule.

Recall the last five rounds of six-round DEAL. The round keys in use are $R_{2}, \ldots, R_{6}$, the input is the pair $\left(x_{1}, y_{1}\right)$ of 64 -bit strings (where $x_{1}=x_{0}$ is the left half of the plaintext and $y_{1}$ is generated in the first round by $\left.y_{1}=y_{0} \oplus E_{R_{1}}\left(x_{1}\right)\right)$, and the output is the ciphertext $\left(x_{6}, y_{6}\right)$. Consider two input/output pairs $\left(\left(x_{1}, y_{1}\right),\left(x_{6}, y_{6}\right)\right)$ and $\left(\left(x_{1}^{\prime}, y_{1}^{\prime}\right),\left(x_{6}^{\prime}, y_{6}^{\prime}\right)\right)$ with $y_{1}=y_{1}^{\prime}, y_{6}=y_{6}^{\prime}, x_{1} \oplus x_{1}^{\prime}=\alpha=x_{6} \oplus x_{6}^{\prime}$ and $\alpha \neq 0$. 
First, we show that two such input/output pairs cannot both exist: Since $y_{1}=y_{1}^{\prime}$ and $y_{6}=y_{6}^{\prime}$, we have $x_{3} \oplus x_{3}^{\prime}=\alpha=x_{5} \oplus x_{5}^{\prime}$. On the other hand, $y_{1}=y_{2}=y_{1}^{\prime}=y_{2}^{\prime}$ and $x_{1} \neq x_{1}^{\prime}$, thus $y_{3}=y_{2} \oplus E_{R_{3}}\left(x_{2}\right) \neq y_{3}^{\prime}$ and hence $E_{R_{4}}\left(y_{3}\right) \neq E_{R_{4}}\left(y_{3}^{\prime}\right)$. If $x_{3} \oplus x_{3}^{\prime}=\alpha$, then $x_{5} \oplus x_{5}^{\prime}=\alpha \oplus E_{R_{4}}\left(y_{3}\right) \oplus$ $E_{R_{4}}\left(y_{3}^{\prime}\right) \neq \alpha$, in contradiction to the above.

Next, we exploit their non-existence to narrow down the number of possibilities for $R_{1}$ and hence $K_{1}$ :

- Choose a fixed value $y_{6} \in\{0,1\}^{64}$ and $2^{64}$ ciphertexts $\left(s, y_{6}\right)$, where $s \in\{0,1\}^{64}$. Let $\left(x_{0}[s], y_{0}[s]\right)$ denote the corresponding plaintexts. Then we expect to find about $2^{63}$ two-sets $\{s, t\} \subset\{0,1\}^{64}$ with $s \neq t$ and $x_{0}[s] \oplus x_{0}[t]=s \oplus t$.

- Check all possible 56-bit DES keys $R$, whether

$$
y_{0}[s] \oplus E_{R}\left(x_{0}[s]\right)=y_{0}[t] \oplus E_{R}\left(x_{0}[t]\right) .
$$

As we have shown above, this is impossible if $R=R_{1}$. Hence, if "=" holds, we know $R \neq R_{1}$, which limits further key search. If $R \neq R_{1}$, the operation $E_{R}:\{0,1\}^{64}$ can be viewed as a random permutation, hence in this case "=" holds with a probability of $2^{-64}$. Given $2^{63}$ such two-sets $\{s, t\}$, we expect to have reduced the possible choices for $R_{1}$ by $50 \%$.

At first, we have $2^{56}$ choices for $R_{1}$, and the attack takes about $2^{63} * 2^{65} * 2=2^{120}$ DES-encryptions to reduce the number of choices for $R_{1}$ down to $2^{55}$. Repeating the attack, we need another $2^{119}$ DES encryptions to reduce the number of choices down to $2^{54}$, another $2^{118}$ DES encryptions to reduce it to $2^{53}, \ldots$, hence we may pin down $R_{1}$ by doing no more than $2^{121}$ DES encryptions. This leaves open $2^{8}$ choices for $K_{1}$.

Now, the complete master key can easily be recovered by exhaustive key search techniques. In the case of DEAL-128, we need $2^{64} * 2^{8}=2^{72}$ trials to find $\left(K_{1}, K_{2}\right)$. For DEAL-192, we need $2^{64} * 2^{64} * 2^{8}=2^{136}$ trials to recover $\left(K_{1}, K_{2}, K_{3}\right)$. Here though, exhaustive key search is not optimal. Since we have found the first round key of a six-round Feistel cipher, recovering the second round key requires a similar attack on a five-round Feistel cipher and hence is even simpler.

Theoretically, this attack is much better than exhaustive key search, and meet-in-the-middle. But due to the huge amount of chosen ciphertexts required, it is quite impractical.

\subsection{A Dedicated Attack for DEAL-192}

Next, we describe another chosen ciphertext attack. This attack takes more time than the previous one, but only needs $2^{32+\tau}$ chosen ciphertexts 
(with $0<\tau \leq 32$ ) instead of $2^{64}$. E.g., for $\tau=0.5$ the keyspace is reduced by about $50 \%$.

Recall the last four rounds of six-round DEAL, using the round keys $R_{3}, R_{4}, R_{5}$, and $R_{6}$. The input to the last four rounds is $\left(x_{2}, y_{2}\right) \in$ $\{0,1\}^{64}$, the output is $\left(x_{6}, y_{6}\right) \in\{0,1\}^{64}$. Consider two input/output pairs $\left(\left(x_{2}, y_{2}\right),\left(x_{6}, y_{6}\right)\right)$ and $\left(\left(x_{2}^{\prime}, y_{2}^{\prime}\right),\left(x_{6}^{\prime}, y_{6}^{\prime}\right)\right)$ with $y_{6}=y_{6}^{\prime}, x_{2} \oplus x_{2}^{\prime}=$ $\alpha=x_{6} \oplus x_{6}^{\prime}$ and $\alpha \neq 0$. (The value $y_{2} \oplus y_{2}^{\prime}$ may be arbitrary.)

Two such input/output pairs cannot both exist: Since $x_{6} \oplus x_{6}^{\prime}=\alpha$ and $y_{6}=y_{6}^{\prime}$, we have $x_{5} \oplus x_{5}^{\prime}=\alpha=x_{4} \oplus x_{4}^{\prime}$, and hence $y_{4} \oplus y_{4}^{\prime} \neq 0$. On the other hand, since $x_{2} \oplus x_{2}^{\prime}=x_{3} \oplus x_{3}^{\prime}=\alpha$, and $x_{5} \oplus x_{5}^{\prime}=\alpha=x_{4} \oplus x_{4}^{\prime}$, we need $y_{3} \oplus y_{3}^{\prime}=0$. This is in contradiction to $y_{4}=y_{3}$ and $y_{4}^{\prime}=y_{3}^{\prime}$.

As above, we exploit the non-existence of such pairs for key recovery purposes:

- Choose a fixed value $y_{6}=y_{6}^{\prime} \in\{0,1\}^{64}$ and $2^{32+\tau}$ different values $s \in\{0,1\}^{64}$, which gives $2^{32+\tau}$ different ciphertexts $\left(s, y_{6}\right)$. Consider two such ciphertexts $\left(x_{6}, y_{6}\right)$ and $\left.\left(x_{6}^{\prime}, y_{6}^{\prime}\right)\right)$ with $x_{6} \neq x_{6}^{\prime}$ and the corresponding plaintexts $\left(x_{0}, y_{0}\right)$ and $\left(x_{0}^{\prime}, y_{0}^{\prime}\right)$.

- For all possible 56-bit round keys $R$, compute $y_{1}=y_{0} \oplus E_{R}\left(x_{0}\right)$ and $y_{1}^{\prime}=y_{0}^{\prime} \oplus E_{R}\left(x_{0}^{\prime}\right)$.

- For all possible 56-bit round keys $S$, compute $x_{2}=x_{1} \oplus E_{S}\left(y_{1}\right)$, and $x_{2}^{\prime}=x_{1}^{\prime} \oplus E_{S}\left(y_{1}^{\prime}\right)$. If

$$
x_{2} \oplus x_{2}^{\prime}=x_{6} \oplus x_{6}^{\prime},
$$

then the key pair $(R, S)$ can be discarded, i.e., $\left(R_{1}, R_{2}\right) \neq(R, S)$. If $(R, S)$ is the wrong key pair, we expect "=" to hold with a probability of $2^{-64}$. Since we have $2^{32+\tau}$ chosen ciphertexts and thus $\left(\begin{array}{c}2^{32+\tau} \\ 2\end{array}\right)$ $\approx 2^{2(32+\tau)} / 2 \approx 2^{64} * 2^{2 \tau-1}$ sets of exactly two ciphertexts, we expect the fraction of the candidates for $\left(R_{1}, R_{2}\right)$ to be discarded to be roughly $1-2^{-2 \tau}$, e.g. roughly $50 \%$ for $\tau=0.5$.

On a first look, the attack seems to require $2^{32+\tau} * 2^{56} * 2^{56}=2^{144+\tau}$ single encryptions. Actually, if either of the round keys $R$ and $S$ is wrong, we expect to find values $x_{2}, x_{2}^{\prime}, x_{6}$, and $x_{6}^{\prime}$ with

$$
x_{2} \oplus x_{2}^{\prime}=x_{6} \oplus x_{6}^{\prime},
$$

after considering less than $2^{33}$ plaintext ciphertext pairs, on the average. No more encryption operations (or decryption operations) are needed to reject the pair $(R, S)$ of round keys. Hence, the expected number of single encryptions is below $2^{33} * 2^{112}=2^{145}$. Since $145>128$, this attack is not useful for DEAL-128. 
On the other hand, the attack actually is useful for DEAL-192, the DEAL-variant with a key size of 192 bit. Note that the attack only narrows down the number of 192 -bit keys from $2^{192}$ to about $2^{192-2 \tau}$, i.e., on the average $8 * 2^{192-2 \tau} / 2$ additional single DES encryption are needed to find the correct key by brute force.

\subsection{The Memory Requirements}

For the attack described in Section 3.1, we need to store about $2^{64}$ plaintexts, each of 128 bit. This requires $2^{71}$ bits of memory - all other memory requirements are negligible, compared to this.

For the attack described in Section 3.2, the attacker apparently has to store all those about $2^{112-2 \tau} 56$-bit keys for the first two rounds, which are not discarded. The correct 56-bit key pair is not discarded, and can be found by further testing. Given a 56-bit key pair $(R, S) \in\left(\{0,1\}^{56}\right)^{2}$, the "further testing" can be done by exhaustively searching $2^{8+8+64}=2^{80}$ 64-bit key triples corresponding to $(R, S)$ to find the correct one. If all $2^{80}$ key triples corresponding to the pair $(R, S)$ are wrong, then $(R, S)$ is wrong, too. Instead of storing the pairs $(R, S)$ and later do the "further testing", one may test immediately and save the storage space.

What then dominates the storage space for the attack described in Section 3.2 is the necessity to store $2^{32+\tau}$ plaintexts, i.e., $2^{39+\tau}$ bits. The attack in Section 3.2 improves on the attack in Section 3.1 both with respect to storage space and to the number of chosen ciphertexts.

Table 1 shows the requirements for the attack in Section 3.2, depending on the parameter $\tau$, i.e., the required number of chosen ciphertexts, the approximate number of single DES encryptions, and the approximate number of storage bits.

Table 1. Requirements for the attack from Section 3.2

\begin{tabular}{c|c|c|c} 
parameter & chosen ciphertexts & single encryptions & memory \\
\hline \hline$\tau$ & $2^{32+\tau}$ & $8 * 2^{191-2 \tau}+2^{145}$ & $2^{39+\tau}$ bit \\
\hline \hline 0.5 & $2^{32.5}$ & $8 * 2^{190}$ & $2^{39.5}$ bit \\
\hline 1 & $2^{33}$ & $8 * 2^{189}$ & $2^{40}$ bit \\
\hline 8 & $2^{40}$ & $8 * 2^{175}$ & $2^{47}$ bit \\
\hline 16 & $2^{48}$ & $8 * 2^{159}$ & $2^{55}$ bit \\
\hline 24 & $2^{56}$ & $8 * 2^{143}+2^{145}$ & $2^{63}$ bit
\end{tabular}




\subsection{Chosen Plaintext Attacks}

The chosen ciphertext attacks we described in Sections 3.1 and 3.2 target on the first round key $R_{1}$ or the first two round keys $R_{1}$ and $R_{2}$. In principle, these attacks do not depend on any key schedule but work well with DEAL-versions with independent round keys. For reasons of symmetry, they can also be run backwards as chosen plaintext attacks, then recovering the last round key or the last two round keys. In fact, the attack described in Section 3.1 can be viewed as the backward version of the chosen plaintext attack on DEAL with independent round keys, described by Knudsen [1]. (The attack in section 3.2 is new, though.)

The reason why we considered chosen ciphertext attacks instead of the possibly more natural chosen plaintext attacks, is the DEAL key schedule. It enables a more simple exploitation of knowing the first or the first two round keys, than knowing the last or the last two ones.

\section{Final Remarks}

\subsection{The Effective Key Size}

From a general point of view, the designer of a new cryptosystem should be pessimistic. I.e., when trying to evaluate the effective key size of a cipher, known standard techniques (such as meet in the middle) should be taken into consideration, even if they appear to be very unrealistic. This defines a safety margin, valuable if new and more practical attacks are found.

Thus, we consider the effective key size of DEAL-128 to be 121 bits (or less) and the effective key size of DEAL-256 to be no more than 224 bits. The effective key size of DEAL-192 is 121 bits (or less). In this sense, DEAL-192 does not improve on DEAL-128. If a variant of DEAL is needed faster than DEAL-256 but with an effective key-size of more than 121 bits, a seven-round version of DEAL would be appropriate.

\subsection{The DEAL Key Schedule}

The DEAL key-schedule is based on using slow but powerful encryption operations. On the other hand, the first round key $R_{1}$ does not depend on all (master-)sub-keys $K_{i}$ (neither does $R_{2}$ depend on all sub-keys of DEAL-192 and DEAL-256, neither does $R_{3}$ depend on all sub-keys of DEAL-256). Once we have recovered $R_{1}$ (and/or $R_{2}, R_{3}$ ), recovering the complete master key is more easy than necessary. Under ideal circumstances, with randomly chosen keys $K_{i} \in\{0,1\}^{64}$, independently and 
according to the uniform probability distribution, this is a minor concern.

But Vaudenay [4] observed the following:

If the choice of the keys $K_{i}$ is restricted, the attacks described in this paper can be improved. Think of the bytes of the keys $K_{i}$ being restricted to a set of $c<2^{7}$ printable characters. This indicates that there are only $c^{8}$ choices for $K_{i}$, and $c^{24}$ choices for a DEAL-192 key. Since the number of choices for $R_{1}$ is reduced to $c^{8}$ instead of $2^{56}$ the attack in Section 3.1 becomes $2^{56} / c^{8}$ times faster. (Note that there is no speed-up for corresponding chosen plaintext attack described by Knudsen [1], since the number of choices for $R_{6}$ still is $2^{56}$.) Similarly, the required number single encryptions for the attack in Section 3.2 is reduced to $8 * c^{24} * 2^{-2 \tau} * 2^{-1}+2^{33} * c^{16}$, instead of $8 * 2^{3 * 64} * 2^{-2 \tau} * 2^{-1}+2^{33} * 2^{2 * 56}$.

If we think of, say, $c=64=2^{6}$, the effective key size of DEAL-192 is reduced to 144 bit. The attack in Section 3.1 would become $2^{8}$ times faster. Depending on $\tau$, the speed-up for the attack in Section 3.2 would be between $2^{16}$ and $2^{48}$.

Also note that if a part of the key is compromised, then the security of DEAL depends on which part is compromised. E.g., if 64 bits of a DEAL-192 key are compromised, the remaining security should still be about the security of DEAL-128. Apparently, this is no problem if the 64 bits of $K_{3}$ are compromised. But if the attacker knows $K_{1}$, she effectively has to attack a five-round variant of DEAL with a 128-bit key, instead of something comparable to six-round DEAL-128.

Our concern regarding the key schedule could easily be fixed, requiring one additional encryption for DEAL-128, two for DEAL-192, and three for DEAL-256, applying the the same general design principle all DEAL key schedules are based on. For DEAL-128, we propose to use $R_{2}, \ldots$, $R_{7}$ as the round keys (and hence throwing away $R_{1}$ after running the key schedule), where

$$
R_{7}:=E_{R_{*}}\left(K_{1} \oplus R_{6} \oplus \underline{5}\right),
$$

where $\underline{5}$ is a constant different from $000 \ldots 0, \underline{1}, \underline{2}, \underline{3}$, and $\underline{4}$. Similar modifications for DEAL-192 and DEAL-256 are obvious.

Note that the additional encryption operations require only between $1 / 6$ and $3 / 8$ of the time for the original key schedule, hence the slow-down for the above modification should be acceptable. 


\subsection{Conclusions and Open Problem}

In spite of these concerns, DEAL is a simple but useful way of constructing a new block cipher based on another block cipher $E$, doubling the block size. There is no limitation to $E=\mathrm{DES}$. One could as well view DEAL as a "mode of operation" of the underlying block cipher $E$, instead of a block cipher of its own right.

One may reasonably expect DEAL to be significantly more secure than its building block DES. It would be interesting to actually prove this, assuming the security of the underlying building block. This has been done before for cryptographic primitives based on other primitives, e.g. for the DES-based 64-bit block cipher DESX [2]. Such a result may seen as a justification of the construction's soundness, though the actual security of the construction also depends on the security of the underlying block cipher.

\section{Acknowledgements}

After discussing the DEAL security with me, Rüdiger Weis was perpetually nagging, waiting for the first version of this paper to be finished. I also thank Don Coppersmith for suggesting an improvement of the attack in Section 3.2 and Richard Outerbridge for discussing the key schedule. I appreciate the comments from the members of the program committee and their help in improving this presentation.

\section{References}

1. L. Knudsen: "DEAL - a 128-bit Block Cipher", February 21, 1998, revised May 15, 1998: http://www.ii.uib.no/ larsr/aes.html.

2. J. Kilian, P. Rogaway: "How to Protect DES Against Exhaustive Key Search", Neal Koblitz (ed.), Advances in Cryptology: Crypto '96, Springer LNCS 1109, 252-267, full version online:

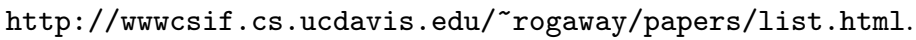

3. P. van Oorschot, M. Wiener, "Improving Implementable Meet-in-the-Middle Attacks by Orders of Magnitude", Crypto '96 Springer LNCS 1109, 229-236.

4. S. Vaudenay, "On Comparing the Security of Block Ciphers", manuscript, 1998. 